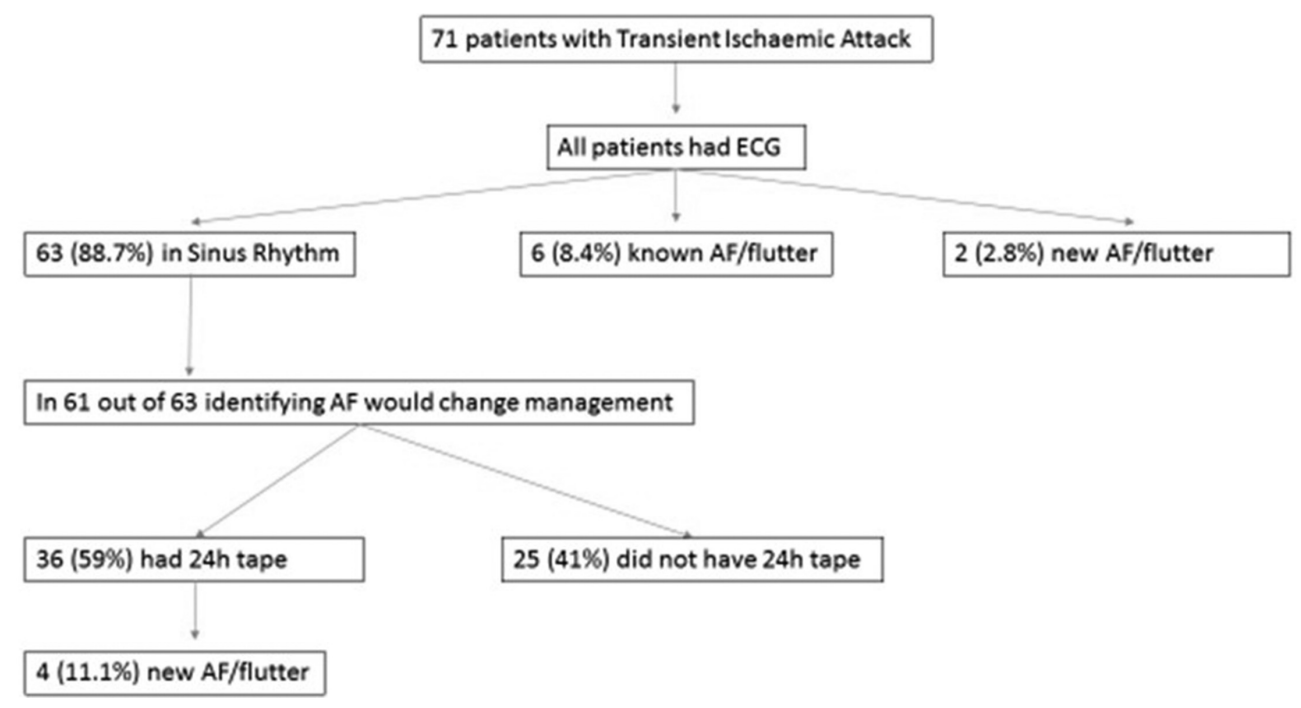

Abstract 42 Figure 2

43 ABSTRACT WITHDRAWN

44 DIAGNOSTIC YIELD OF 7-DAY HOLTER ECG MONITORING FOR NEW AF AND ASSOCIATION WITH COMORBIDITIES. OUTCOMES IN AN ALL-COMER COHORT FROM A LARGE TEACHING TRUST

${ }^{1}$ Muhammad Jawad UI Qamar* ${ }^{2}$ Yanish Purmah, ${ }^{3}$ Muhammad Nawaz, ${ }^{1}$ Paulus Kirchhof. ${ }^{1}$ University of Birmingham centre for Cardiovascular Research, SWBH Trust; ${ }^{2}$ Queen Elizabeth Hospital, University Hospital Birmingham; ${ }^{3}$ Sndwell and West Birmingha Hospital NHS Trust

10.1136/heartjnl-2017-311726.43

Introduction Prolonged ECG monitoring is an increasingly advocated method to detect silent AF and other arrhythmias. Inevitably, the wider use of prolonged ECG monitoring will yield unexpected and serious findings requiring swift action.
Abstract 44 Table 1

\begin{tabular}{|l|c|c|c|c|}
\hline $\begin{array}{l}\text { Indication for } 7 \\
\text { day event } \\
\text { monitoring }\end{array}$ & \multicolumn{2}{|c|}{ New AF } & \multirow{2}{*}{ Total } & $\begin{array}{c}\text { Pick-up } \\
\text { Pescentage }\end{array}$ \\
\cline { 2 - 3 } Palpitations & 16 & No & & Percent \\
Dizziness & 0 & 51 & 51 & $9.8 \%$ \\
Syncope & 0 & 31 & 31 & $0 \%$ \\
Chest Pain & 0 & 10 & 10 & $0 \%$ \\
CVA & 9 & 39 & 48 & $18.7 \%$ \\
Total & 25 & 293 & 318 & $7.8 \%$ \\
\hline
\end{tabular}

Here, we report the diagnostic yield of 7 day Holter ECG monitoring requested through primary and secondary care. We also highlight the clinical and Holter ECG parameters associated with new AF.

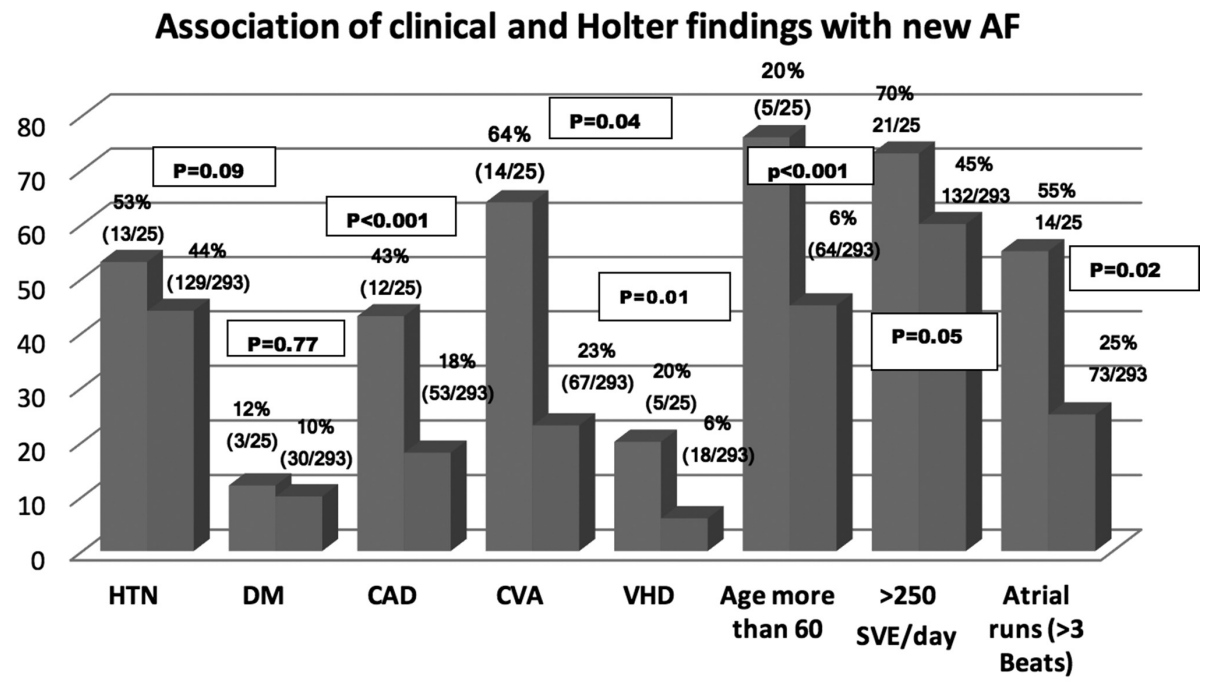

New AF $(n=25) \quad$ No AF $(n=293)$ 
Methods We analysed all 7 day Holter ECG tests in Sandwell and West Birmingham NHS Trust from 1st April 2014 to 30th April 2016, using Spacelabs Sentinel server running Pathfinder V 1.7.1. Tests were bookable by primary care and hospital physicians. Holter recordings from 364 patients, previously reported by cardiac physiologists were reviewed by physicians trained in Holter analysis. 46 patients had previously diagnosed AF and were excluded from the analysis. Here we present the results from a final cohort of 318 patients.

Results Mean age of the patients was 59.2 years (median 56 years, IQR 43-72) and 55\% were male. Average time of monitoring was 162.2 hours. Palpitations (56\%), dizziness (16\%), ischaemic stroke or TIA (15\%), syncope (10\%), and chest pain $(3 \%)$ were reasons for referral. New AF was diagnosed in 25/318 patients. The pick-up rate of incidental AF was $7.86 \%$. 9/48 (18.7\%) of patients with CVA and 16/178 (8.9\%) with palpitations had new AF. For 7 day Holters performed with intention to look for AF (stroke and palpitations) the combined diagnostic yield to pick up new AF was $11 \%$. There was no AF detected for indications of dizziness, syncope or chest pain.

Patients with newly diagnosed AF were older with mean age 71 years (CI 66-78, SD 17.3) vs. 57 years (CI 55-59, SD 17.6) for non-AF group $(\mathrm{p}=0.001)$, more often suffered from coronary artery disease $(43 \%$ vs $18 \%, \mathrm{p}=0.001)$, prior stroke or TIA (64\% vs $23 \%, \mathrm{p}=0.04)$, moderate to severe valvular heart disease $(20 \%$ vs $6 \%, p=0.01)$, had higher occurrence of $>250$ supra ventricular ectopics/day $(73 \%$ vs $60 \%, \mathrm{p}=0.05)$ and atrial runs of more than 3 beats $(55 \%$ vs $25 \%$, $0=0.002$ respectively). Heart rate parameters for patients with and without AF were not different in our cohort. Other medically relevant findings were 2nd degree AV block (9/318, $2.8 \%)$, 3rd degree AV block $(2 / 318,0.6 \%)$ and non-sustained VT $(11 / 318,3.4 \%)$.

Conclusions Prolonged ECG monitoring in a real life cohort, especially in patients with high risk of AF, leads to higher diagnostic yield, with significant therapeutic consequences. It appears reasonable to provide open access to prolonged ECG monitoring to at-risk populations in primary and secondary care. Further analysis of risk factors for paroxysmal AF and systematic evaluations of treatment and outcomes after AF screening are warranted to define populations for screening for silent $\mathrm{AF}$.

\section{RIVAROXABAN VS. DABIGATRAN OR WARFARIN IN REAL-WORLD OBSERVATIONAL STUDIES OF STROKE PREVENTION IN ATRIAL FIBRILLATION: SYSTEMATIC REVIEW AND META-ANALYSIS} ${ }^{1}$ Ying Bai*, ${ }^{2}$ Hai Deng, ${ }^{3}$ Alena Shantsila, ${ }^{4}$ Gregory YH Lip. ${ }^{1}$ Beijing Tongren Hospital;
${ }^{2}$ Guangdong General Hospital, Guangdong Academy of Medical Science; ${ }^{3}$ University of
Birmingham, Institute of Cardiovascular Sciences,City Hospital; ${ }^{4}$ University of Birmingham,
Institute of Cardiovascular Science, City Hospital

\subsection{6/heartjnl-2017-311726.44}

Objective To evaluate the effectiveness and safety of rivaroxaban in real-world practice compared with dabigatran ( $\mathrm{R}$ vs. $\mathrm{D}$ ) or with warfarin ( $\mathrm{R}$ vs. W) for stroke prevention in atrial fibrillation (AF).

Methods and Results 17 studies were included after a thorough search in PubMed for studies reporting comparative effectiveness and safety of R vs. D(3), R vs. W (11) or both conditions (3)for stroke prevention in AF. Overall, the risks of ischaemic stroke (IS)/systematic thromboembolism (TE) with rivaroxaban were similar compared with dabigatran (Figure 1) IS/TE: (HR:1.02,95\% CI: 0.91-1.13, I2=70.2\%, $\mathrm{n}=5$ ), but were significantly reduced when compared to warfarin. IS/TE: $(0.75,0.64-0.85, \mathrm{I} 2=45.1 \%, \mathrm{n}=9)$. Major bleeding risk was significantly higher with rivaroxaban than dabigatran (Figure 2) $(1.38,1.27-1.49, \mathrm{I} 2=26.1 \%, \mathrm{n}=5)$, but similar to warfarin $(0.99,0.91-1.07, \mathrm{I} 2=0.0 \%, \mathrm{n}=6)$. Rivaroxaban was associated with increased all-cause mortality and gastrointestinal bleeding (GIB), but similar risk of acute myocardial infarction (AMI) and intracranial bleeding (ICB) compared with dabigatran. When compared with warfarin, rivaroxaban was associated with similar risk of any bleeding, mortality and AMI, but a higher risk of GIB and lower risk of ICB.

Conclusions In this systematic review and meta-analysis, rivaroxaban was as effective as dabigatran, but was more effective

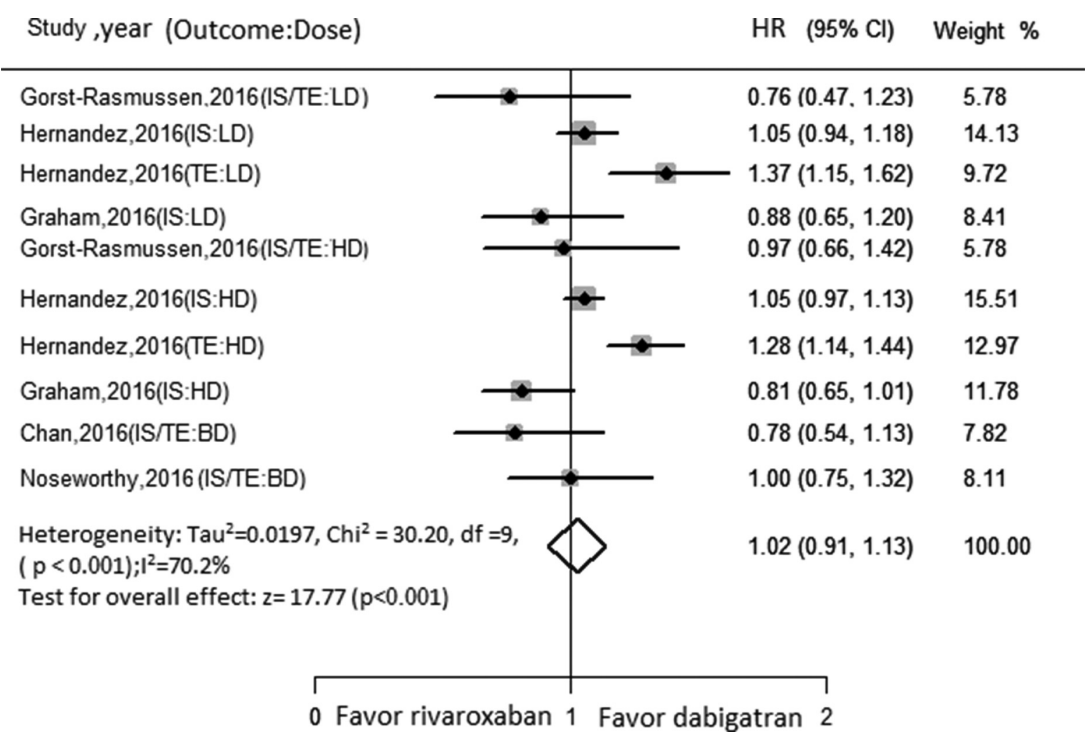

Abstract 45 Figure 1 Rivaroxaban compared with Dabigatran in risk of IS/TE in AF patients. IS, ischemic stroke, TE, thromboembolism. LD,lowdose; HD, high-dose; $B D$, both-dose. $A F$, atrial fibrillation 